O. O. Biloborodov, Cand. Sc. (Eng.)

\title{
STUDY OF THE ORIENTATION ERRORS IMPACT ON THE ACCURACY OF GUIDANCE
}

Separate samples of the engineering systems influence different objects without the self- control regarding the accuracy of the impact. From technical point of view this implies the need to substantiate the requirements concerning the accuracy of the guidance and performing the studies of the factors, influencing the accuracy characteristics of the guidance systems. If we consider the dependence of the guidance errors ( in the azimuth and elevation angle) on the orientation errors, then their functional dependence will be determined. In such case it is not correct to apply the formula for the determination of the root-mean-square deviation of the sum of the independent random values and there appears the task of determining the impact of such class of errors in the general errors and determination of the possible ways of their minimization. The results of the study of the orientation errors impact on the guidance accuracy of the directed system are presented. For emitting radioengineering systems referencing and orientation errors are approximately $60 \%$ of the guidance errors. The given research shows the heterogenous character of the orientation errors impact (both by the value and by the degree of the impact) on the guidance errors. Analytical study of the guidance errors in the multidimensional area of arguments has some problems, taking into consideration large volume of the analytical expressions and multidimensional character of the dependences. The results of the simulation experiment of determining the character of the orientation errors impact on the indices of the guidance errors are submitted. It was determined that the guidance errors also depend on the target direction. The study of the guidance errors in the area of the value of the possible target directions was performed. The availability of the areas of minimal and maximal guidance errors in the area of the azimuth and elevation angle values was determined, the location of these areas depend on the relation of the orientation errors values. For the application of the facilities of the directed action the usage of the method of timely rational orientation of the carrier for the reduction of the guidance errors is suggested.

Key words: orientation, guidance errors, functional dependence, simulation experiment.

\section{Introduction}

Problem set-up. Separate samples of the engineering systems influence various objects without the self- control regarding the accuracy of the impact. The examples of such systems could be laser systems of various designation or transmitting radioengineering systems (radioelectronic combat or others). Network centric technologies often provide the separation of the functions of detecting the objects of the impact and direct impact on them. For such systems the important task is the accurate guidance on the object of impact by means of recalculation of the coordinates of the object of impact (as a rule, these are the azimuth and elevation angle). High requirements regarding the selectivity of the action are put forward to the new samples of radioengineering equipment [1]. From the technical point of view this implies the necessity to substantiate the requirements regarding the accuracy of the guidance and perform the studies of the factors influencing the accuracy characteristics of the guidance systems.

Analysis of the recent research and publications. When the target location is determined by means of scanning, the accuracy of the angular coordinates determination depends on the width of the directional pattern [2]. But modern means of the impact are developed, in particular, as a result of the introduction of the indiscriminate guidance systems [3]. The growth of the dimensions of the antenna systems enables to increase the operating range, accuracy, angular discrimination performance; at the same time the construction of the antennas becomes more complicated [4]. Mechanical errors of assembling systems also influence greatly the accuracy of the guidance and tracking [5]. Determination of the guidance accuracy is the function of the complex technicaleconomic compromise [6]. The construction of the guidance systems, besides the requirements 
concerning accuracy often must meet the requirements of the reliability and operation efficiency [7]. The researchers study the problem of the impact of the navigation data accuracy on the results of the systems operation [8]. At the same time, insufficient attention is paid in the studies of the universal methods and program-algorithmic means, intended for the assessment of the numerical characteristics of the quality indices worsening of systems (complexes) usage as a result of the errors of the orientation determination subsystems.

For the radiolocation equipment the accuracy of the determination of the target coordinates is characterized mainly by the accuracy of the measurements: directional pattern width, signal/noise ratio, number of frequency bursts of direct impulses, fluctuations errors of the reflected signal amplitude, etc.) [9]. Orientation errors are considered to be independent and represent not more than $10 \%$ of the total errors of the angular definitions. For passive systems or radioengineering systems without the feedback the mechanical errors represent not more than $40 \%$ and the rest are the errors of the reference and orientation. The components $\sigma_{i}$ of the total error $\sigma_{\Sigma}$ are considered to be independent and mean square deviation of the total error is calculated by the classic formula:

$$
\sigma_{\Sigma}=\sqrt{\sigma_{i}^{2}}
$$

However, if we consider the dependence of the guidance errors (by the azimuth and elevation angle) on the orientation errors, its functional dependence will be revealed. In such case it is incorrect to use (1) and there appears the problem of determining the impact of such class of errors on the general errors and determining the possible ways of their minimization.

Taking into account the above-mentioned, the aim of the paper is to determine the character of the impact of the orientation errors of the basic carrier (platform) on the guidance errors of the directed system.

\section{Methods of studies}

The following methods were used in the research: method of the uniform coordinates - for basic geometric transformations of the revolution and shift; geometric methods for the determination of the trigonometric functions of the components of the guidance vector across the coordinates of the target and base as well as base orientation; methods of the functional analysis and functions linearization - for the analytical determination of the form of function of mean square deviation (MSD) of the errors of the guidance vector components; methods of the mathematical simulation and statistical analysis - for carrying out the program experiment of the study of the orientation errors impact on the guidance errors; decomposition method - for studying the impact of separate parameters on the guidance errors in multidimensional space of impact arguments; projections methods - for the analysis of the results of the guidance errors study.

\section{Main part}

In general case the position of the object of influence in the space is determined by the extraneous facilities (coordinates of the target, boundaries of the area of influence). Navigation system determines the coordinates and orientation of the influence facility carrier (base). Computation system of the base according to the determined data, defines the necessary parameters of the guidance (azimuth $\theta$ and elevation angle $\phi$ ) and the system (complex) guides the antenna system in the corresponding direction .

\section{Determination of the pointing angles}

We will consider the order of the guidance vector determination $(\theta, \phi)$. By means of the basic system of the coordinates we select the geocentrical (greenwich) rectangular coordinates system 
[10]. The specimen (carrier of the antenna system) determines its coordinates $B^{C K 0}\left(x_{B}, y_{B}, z_{B}\right)$, orientation $R^{C K 0}(\alpha, \beta, \gamma)$, and also obtains the coordinates of the target $C^{C K 0}\left(x_{C}, y_{C}, z_{C}\right)$. Further the specimen determines the guidance vector that comprises the azimuth $\theta$ and elevation angle $\phi$. We will introduce CS, connected with the antenna system (CS1): center of the coordinates (p. $A$ ) - phase center of the antenna; axis $A x^{\prime}$ - is directed along the electric axis of the antenna; axis $A y^{\prime}-$ in the plane of the antenna aperture, perpendicular to the symmetry plane, that passes across the geometric center of the antenna and geometric center of the irradiator; axis $A z^{\prime}$ supplements the system to the right.

The control parameters of the guidance subsystem should be found: such rotation angles of CS1 relatively $\mathrm{CS} 0$, that axis $A x^{\prime}$ be directed to the target. For this purpose it is sufficient to find the coordinates of the vector $\overrightarrow{A C}$ (in CS1), and find angles $\theta, \phi$ for the coincidence of the axis $A x^{\prime}$ with $\overrightarrow{A C}$.

The coordinates of the target in CS1 can be found by means of the known coordinates of the beginning of CS1 in CS0 and the known orientation of CS1 relatively CS0. The corresponding coordinates are determined by the expression:

$$
C^{\prime}\left(x_{C}^{\prime}, y_{C}^{\prime}, z_{C}^{\prime}\right)=C\left(x_{C}, y_{C}, z_{C}\right) \cdot\left\|A^{C^{\prime} \leftarrow C}\right\|,
$$

where the transfer matrix equals:

$$
\left\|A^{C^{\prime} \leftarrow C}\right\|=\left\|A_{\Delta L}^{C^{\prime} \leftarrow C}\right\| \cdot\left\|A_{\llcorner}^{C^{\prime} \leftarrow C}\right\|,
$$

and the components of the transfer matrix (shift matrix $\left\|A_{\Delta L}^{C^{\prime} \leftarrow C}\right\|$ and rotation matrix $\left\|A_{\llcorner}^{C^{\prime} \leftarrow C}\right\|$ ) are determined according to the set of the homogeneous coordinates [11].

Further coordinates are presented into CS1. For the coincidence of the axis $A x^{\prime}$ with $\overrightarrow{A C}$ the rotation around the axis $A y^{\prime}$ and then around axis $A z^{\prime}$ should be performed (Fig. 1). To determine the rotation angle $\theta$ around the axis $A y^{\prime}$ (for the transition of $\overrightarrow{A C}$ into the plane $A x^{\prime} y^{\prime}$ ) $\overrightarrow{A C}$ first will be projected on the plane $A x^{\prime} z^{\prime}$. The length of the projection:

$$
d=\sqrt{x_{C}^{2}+z_{C}^{2}} .
$$

Trigonometric functions of the angle $\theta$ :

$$
\begin{aligned}
& \sin \theta=-\frac{z_{C}^{\prime}}{\sqrt{x_{C}^{\prime 2}+z_{C}^{\prime 2}}}, \\
& \cos \theta=\frac{x_{C}^{\prime}}{\sqrt{x_{C}^{\prime 2}+z_{C}^{\prime 2}}},
\end{aligned}
$$

where the negative sign while $\sin \theta$ determining is caused by the negative direction of the rotation (clockwise).

After transfer into the plane $A x^{\prime} y^{\prime}$ by the means of the rotation around the axis $A y^{\prime}, x^{\prime}$ the component of the vector $\overrightarrow{A C}$ will equal $d$, and $y^{\prime}$ component will equal $\left(y_{C}-y_{A}\right)$. Thus, the trigonometric functions of the rotation angle $\phi$ around the axis $A z^{\prime}$, needed for the coincidence of $\overrightarrow{A C}$ with the axis $A x^{\prime}$, equal:

$$
\sin \phi=\frac{y_{C}}{|\overrightarrow{A C}|}=\frac{y_{C}^{\prime}}{\sqrt{x_{C}^{\prime 2}+y_{C}^{\prime 2}+z_{C}^{\prime 2}}},
$$




$$
\cos \phi=\frac{d}{|\overrightarrow{A C}|}=\frac{\sqrt{x_{C}^{\prime 2}+z_{C}^{\prime 2}}}{\sqrt{x_{C}^{\prime 2}+y_{C}^{\prime 2}+z_{C}^{\prime 2}}} .
$$

Thus, the guidance vector is defined by means of $(6)-(8)$ :

$$
\theta=\operatorname{arctg}\left(\frac{\sin \theta}{\cos \theta}\right), \phi=\operatorname{arctg}\left(\frac{\sin \phi}{\cos \phi}\right),
$$

where the quadrant $\theta$ and $\phi$ are determined, taking into account the signs of the sines and cosines of the corresponding angles.

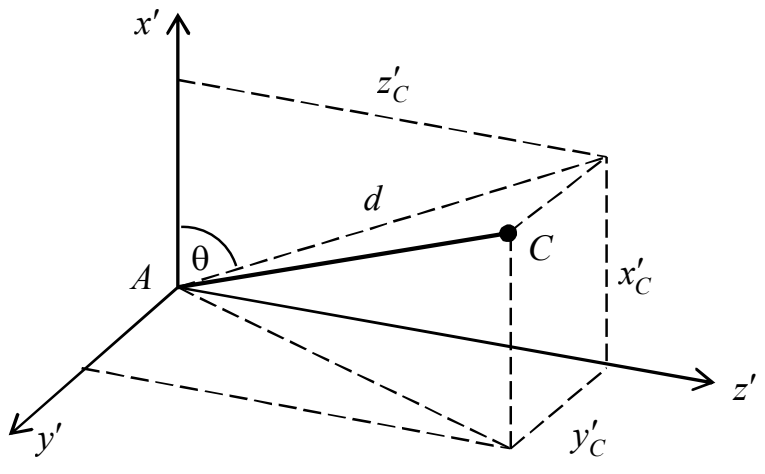

a)

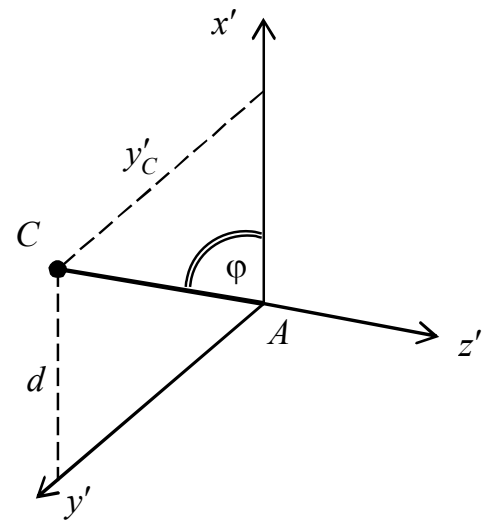

b)

Fig. 1. Rotations for the coincidence: a) initial position; b) after the rotation around the axis $A y^{\prime}$

Hence, the task of the guidance is to determine the corresponding guidance angles (guidance vector) according to (9).

The coordinates of the target are determined by the external means, that is why, the coordinates errors of the target are not considered. In (9) variables $\left(\tilde{x}_{B}, \tilde{y}_{B}, \tilde{z}_{B}, \tilde{\alpha}_{R}, \tilde{\beta}_{R}\right.$ and $\left.\tilde{\gamma}_{R}\right)$, on which functions $(\theta$ and $\phi)$ depend on, are of probabilistic character with random errors: $\tilde{x}_{B}=x_{B}+\delta x$, $\tilde{y}_{B}=y_{B}+\delta y, \quad \tilde{z}_{B}=z_{B}+\delta z ; \quad \tilde{\alpha}_{R}=\alpha_{R}+\delta \alpha, \quad \tilde{\beta}_{R}=\beta_{R}+\delta \beta, \quad \tilde{\gamma}_{R}=\gamma_{R}+\delta \gamma$. As a result of errors introduction, we obtain the resulting guidance vector in the form of the mixture of their ideal components with the errors: $\tilde{\theta}=\theta+\delta \theta, \tilde{\phi}=\phi+\delta \phi$. It can be assumed, that the orientation errors are distributed according to the centered normal law with the known MSD. For modern satellite radionavigation equipment, for instance, MSD of the base coordinates determination errors does not exceed $10 \mathrm{~m}$ (it depends on the method and time of determination). MSD of the orientation errors of modern equipment is:

1) multibase phase satellite navigation determinations $-(1.4-3.8) \cdot 10^{-3} \mathrm{rad}$ (size of the base - up to $1.5 \mathrm{~m})[12]$;

2) gyrocompasses $-(0.5-9) \cdot 10^{-4} \mathrm{rad}$;

3) magnetic compasses - up to $9 \cdot 10^{-3} \mathrm{rad}[13]$.

Direct determination of the numerical characteristics of the random functions (9) by the numerical characteristics (NC) of the random arguments can not be realized as a result of nonlinear character of the functions. For the approximate calculation of $\mathrm{NC}$ in this case linearization in the section of mathematical expectation of the arguments is performed. Values of the functions will be written in the form $\theta=f\left(s_{i}\right), \phi=g\left(s_{i}\right)$, where $s=\left(X_{B}, Y_{B}, Z_{B}, \alpha_{R}, \beta_{R}, \gamma_{R}\right)$. Mathematical expectation of the functions (9) will equal to the guidance parameters in case of errors absence. The error dispersion [14]: 


$$
\begin{aligned}
& \sigma_{\theta}^{2}=\sum_{i=1}^{6}\left[f_{s_{i}}^{\prime}\left(s_{1}, . ., s_{6}\right)\right]^{2} \sigma_{i}^{2}=\left[\operatorname{arctg}_{X_{B}}^{\prime}\left(\frac{\sin \theta}{\cos \theta}\right)\right]^{2} \sigma_{X_{B}}^{2}+\ldots+\left[\operatorname{arctg}_{\gamma_{R}}^{\prime}\left(\frac{\sin \theta}{\cos \theta}\right)\right]^{2} \sigma_{\gamma_{R}}^{2}, \\
& \sigma_{\phi}^{2}=\sum_{i=1}^{n}\left[g_{s_{i}}^{\prime}\left(s_{1}, . ., s_{6}\right)\right]^{2} \sigma_{i}^{2}=\left[\operatorname{arctg}_{X_{B}}^{\prime}\left(\frac{\sin \phi}{\cos \phi}\right)\right]^{2} \sigma_{X_{B}}^{2}+\ldots+\left[\operatorname{arctg}_{\gamma_{R}}^{\prime}\left(\frac{\sin \phi}{\cos \phi}\right)\right]^{2} \sigma_{\gamma_{R}}^{2} .
\end{aligned}
$$

Within the frame of the given research we restrict ourselves to the study of the orientation errors. Also it should be noted that besides the errors (10), (11) the errors of the mechanics, mentioned above, should be taken into account (dynamic error of the sensor angel/code, error of mechanics in the process of the sensor installation on the rotation axis, etc).

\section{Study of the character of the absolute values of the guidance errors change}

For the verification of the assumption regarding the heterogeneous character of the orientation errors impact on the guidance errors $\delta \theta$ and $\delta \phi$ the preliminary research was carried out. By means of (9) the guidance vectors in the conditions of orientation errors absence were determined. After that the errors which changed in eight stages according to Table 1 and Fig. 2. were introduced and the guidance vectors in case of errors presence were determined .The difference that is the value of the absolute angular guidance error, showed complex character of the dependence, presented in Fig. 3.

Table 1

\section{Order of the orientation errors changes}

\begin{tabular}{|c|c|c|c|}
\hline № e/c & $\delta \alpha$ & $\delta \beta$ & $\delta \gamma$ \\
\hline 1. & $0 \rightarrow \delta \alpha_{\max }$ & 0 & 0 \\
\hline 2. & 1 & $0 \rightarrow \delta \beta_{\max }$ & 0 \\
\hline 3. & $\delta \alpha_{\max } \rightarrow 0$ & 1 & 0 \\
\hline 4. & 0 & 1 & $0 \rightarrow \delta \gamma_{\max }$ \\
\hline 5. & $0 \rightarrow \delta \alpha_{\max }$ & 1 & 1 \\
\hline 6. & 1 & $\delta \beta_{\max } \rightarrow 0$ & 1 \\
\hline 7. & $\delta \alpha_{\max } \rightarrow 0$ & 0 & 1 \\
\hline 8. & 0 & 0 & $\delta \gamma_{\max } \rightarrow 0$ \\
\hline
\end{tabular}

The results are presented in the form of the absolute value of errors according to the phase paths (Fig. 2) in the orthogonal polar plane with counter --clockwise sense of rotation (Fig. 3), where $\alpha(\beta, \gamma): 0 \rightarrow 1$ means the change of the corresponding error from zero to the maximum value, and $\alpha(\beta, \gamma): 1 \rightarrow 0$ - from the maximum value to zero. In each $45^{0}$ octant the values of two components of the error are fixed and the value of the third error changes from zero to the maximum value or vice versa.

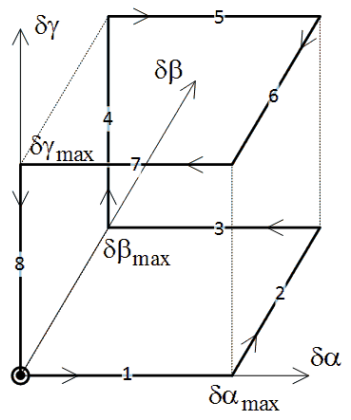

Fig. 2. Phase path of the orientation errors change 
The analysis of the results obtained (Fig. 3) showed the heterogeneous character of the orientation errors impact (both by the value and by the degree of the impact) on the guidance errors, that requires the additional studies.

If the transition matrix is used for the analytic representation of the guidance vector components and after the application of the four-step rule for finding the derivative of the composite function by partial variable the analytical expression for the determination of the azimuth error (10) contains more than 220 components in the form of the trigonometric and fractional-rational functions and the expression for the determination of the error by the elevation angle (11) - more than 430 components in the form of the trigonometric power and fractional rational functions. Analytical study of the guidance errors in multidimensional plane of the arguments is rather difficult, that is why, the realization of the model experiment for the determination of the character of the orientation errors impact on the indices of the guidance errors became the next stage of the research.

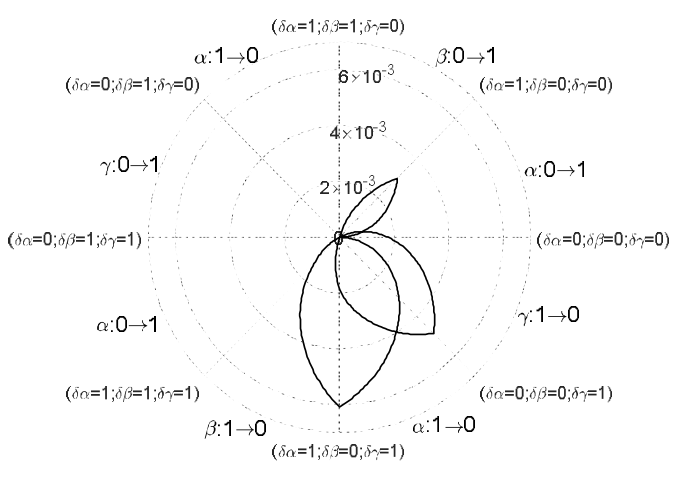

a)

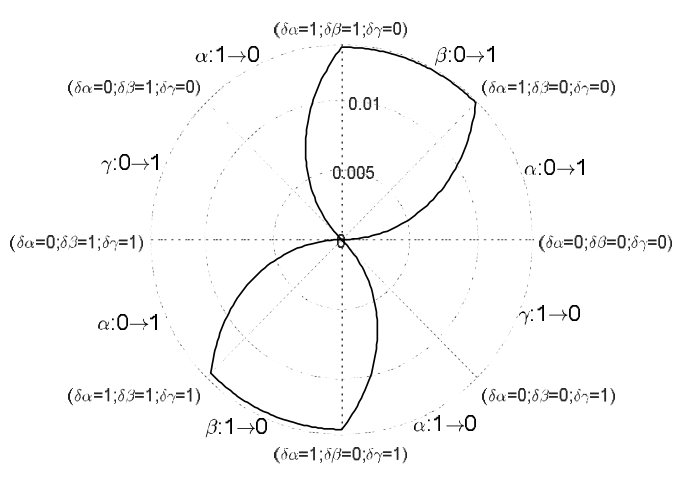

c)

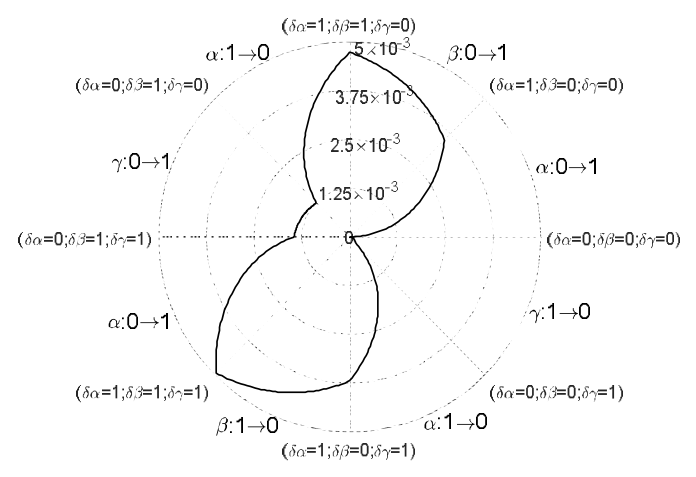

b)

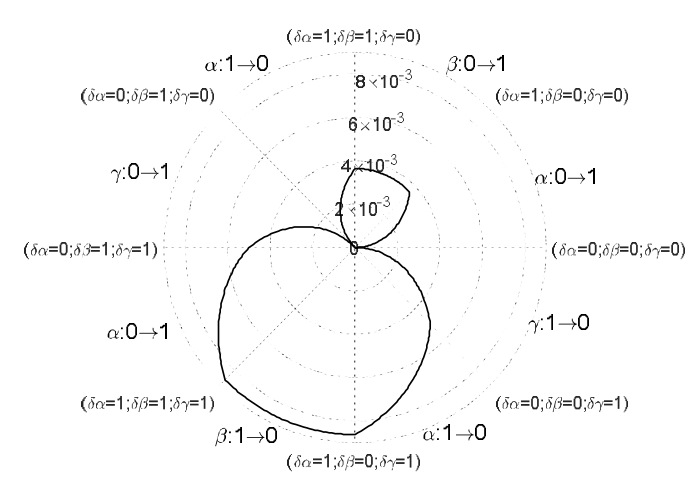

d)

Fig. 3. Example of the guidance errors change: by the azimuth $\delta \theta(a, b)$ and elevation angle $\delta \phi(b, d)$ for various directions: $\theta=45^{\circ}, \phi=45^{\circ}(\mathrm{a}, \mathrm{b}) ; \theta=90^{\circ}, \phi=270^{\circ}(\mathrm{c}, \mathrm{d})$

\section{Model experiment}

For performing the model experiment the following algorithm was developed: serial change of the guidance parameters values (discrete of the guidance vector) was organized ; for each discrete of the guidance vector the target coordinates were determined; further in 3D space MSD of the orientation vector errors 100 tests were performed for a specific set of errors, which were distributed by the centered normal law with MSD, selected at the given step; by the results of the test the parameters of the guidance errors distribution were determined namely: - MSD of the azimuth and elevation angle errors); after the change of MSD of orientation errors the cycle is repeated. Thus, 
MSD of the errors components $\sigma_{\alpha}, \sigma_{\beta}$ and $\sigma_{\gamma}$ was distributed according to the centered normal law with the deviation value from $5 \cdot 10^{-5} \mathrm{rad}\left(\sim 10\right.$ ang. sec) to $5 \cdot 10^{-3} \mathrm{rad}(\sim 17,2$ ang. min.), and discretes of the guidance vector changed in the ranges: $\theta \subset[0 ; 2 \pi], \phi \subset[0 ; 2 \pi]$. Taking into account the complexity of the visualization of the function of three variables, for the presentation of the results the method of color projection on 3D area of the input arguments was selected (Fig. 4).

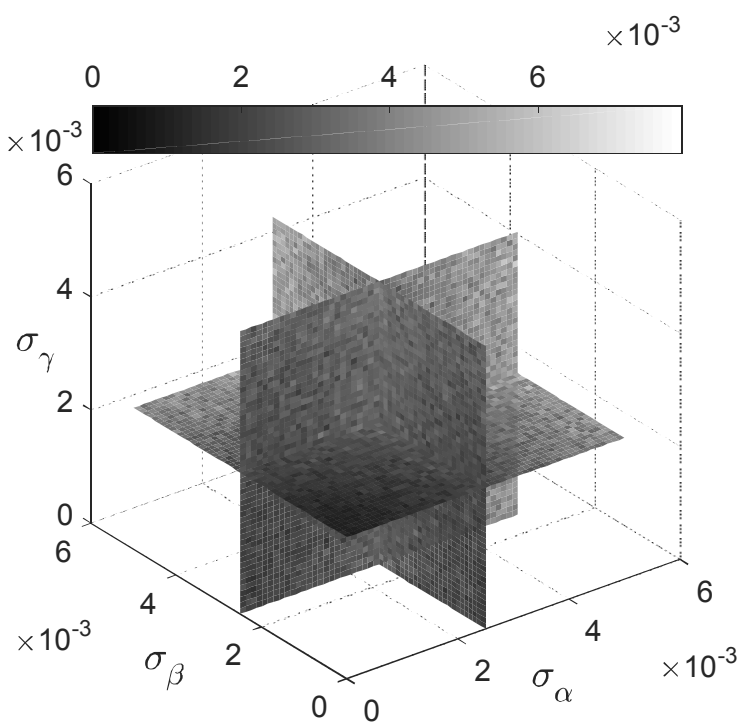

a)

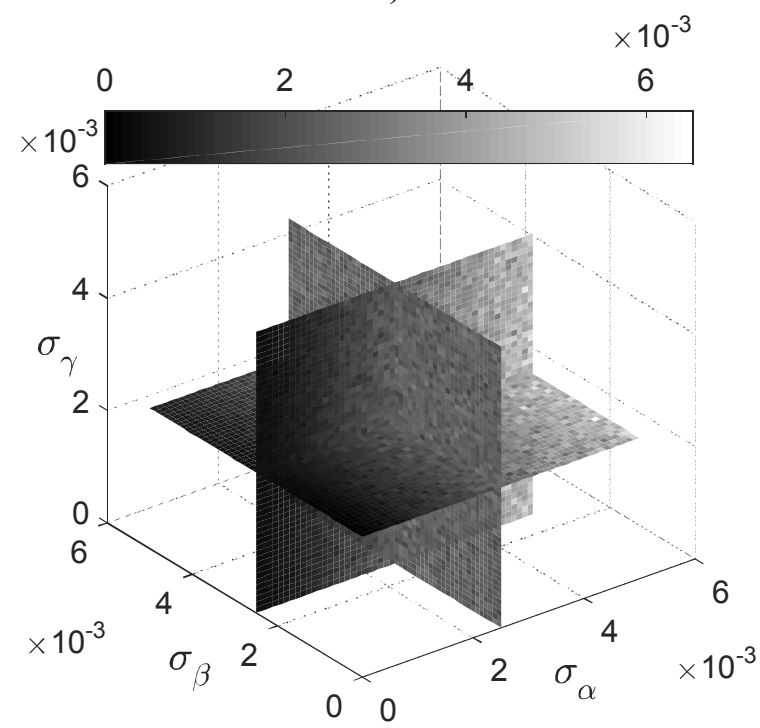

c)

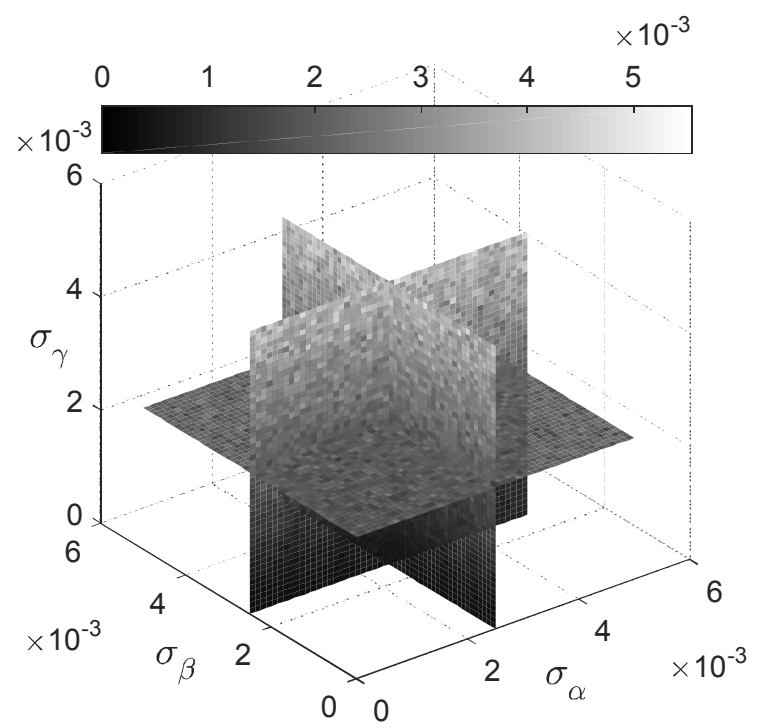

b)

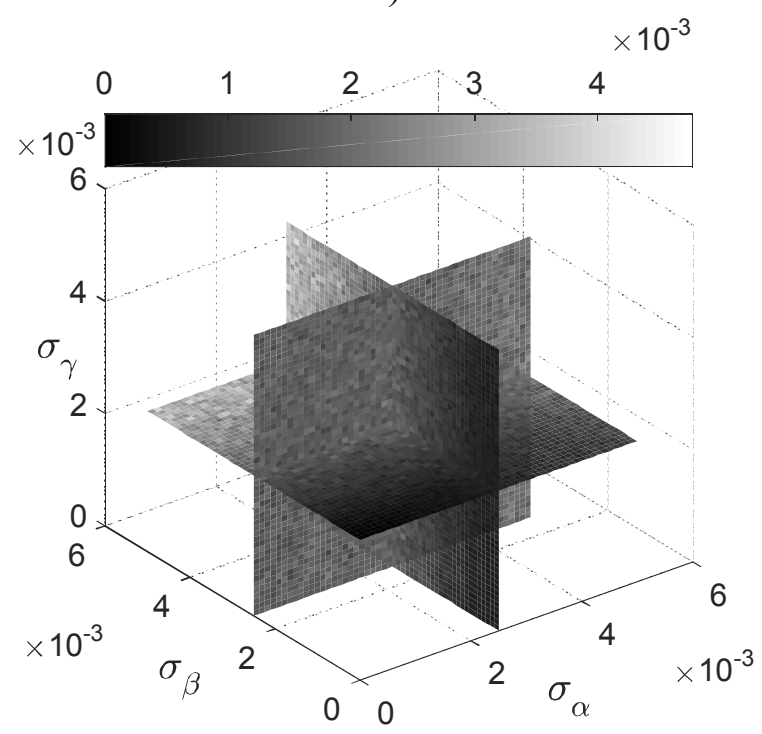

d)

Fig. 4. Separate results of the model experiment for the determination of MSD of the guidance errors distribution by the azimuth $\sigma_{\theta}(\mathrm{a}, \mathrm{c})$ and by the elevation angle $\sigma_{\phi}(\mathrm{b}, \mathrm{d})$ depending on MSD of the orientation errors for various directions: $\theta=90^{\circ}, \phi=225^{\circ}(\mathrm{a}, \mathrm{b}) ; \theta=315^{\circ}, \phi=180^{\circ}(\mathrm{c}, \mathrm{d})$

Analysis of the results of model experiment allowed to make the following conclusions:

for various pairs of values of azimuth - elevation angle at the same values of the input errors different by the magnitude values of the dispersion of the resulting guidance errors are observed; non-linear character of the guidance errors dependence on the orientation errors is observed;

in separate points of the azimuth values and elevation angle area the instability of the solution of the guidance errors search problem is determined: considerable (up to several orders) surges of the Scientific Works of VNTU, 2020, № 2 
dispersion values of the obtained errors are detected.

\section{Study of the guidance errors dependence on the targetdirection}

For studying the dependence of the guidance errors on the target direction the method of fixation of the value of the guidance errors dispersion and the study of the guidance errors in the area of the possible target directions value was chosen. For this purpose the analytical expressions for (10) and (11) were presented in the form of the programming modules of the calculation of the values of guidance errors dispersion. Thus, although the direct functional analysis (10) and (11) is difficult to perform, however, they can be used for separate studies.

At the first stage the periodic character of the errors change as well as availability of the sections of maximum and minimum MSD values of the guidance errors was determined (Fig. 5).

Also the character of errors changes at various values of the orientation vector errors components was studied (Fig. 6)

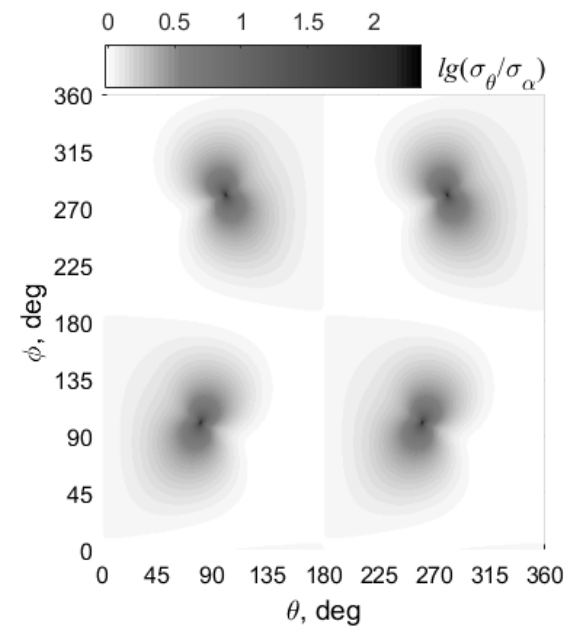

a)

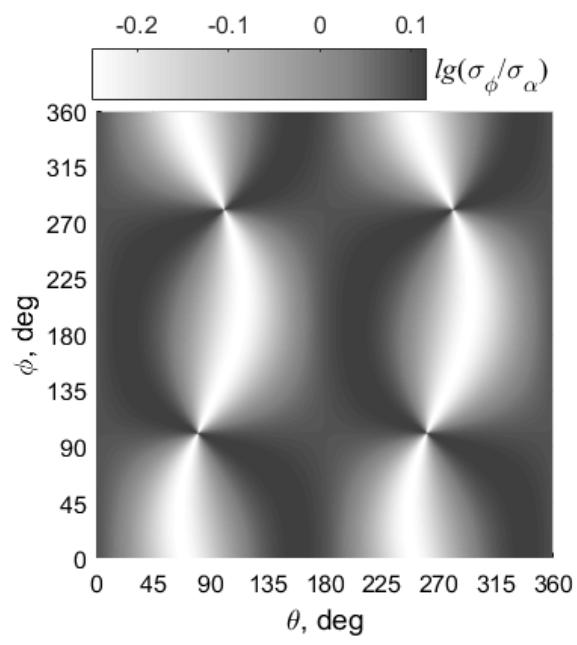

b)

Fig. 5. Results of the study of the periodicity and MSD of the guidance errors by the azimuth (a) and elevation angle (b), depending on the target direction for $\sigma_{\alpha}=\sigma_{\beta}=\sigma_{\gamma}=5 \cdot 10^{-3} \mathrm{rad}(\sim 17.2$ ang. min.)

Analysis of the obtained results allowed to make the following conclusions:

- Extremum for MSD of the azimuth errors and elevation angle (has the form of the point of inflection), is located near the direction $(\theta, \phi) \approx\left(80^{\circ}, 100^{\circ}\right)$;

- The presence of minimal and maximal guidance errors areas in azimuth and elevation angle values area is determined, the location of these areas depends on the ratio of orientation errors values.

The numerical search for MSD extrema of the errors for different values of MSD of the orientation errors was performed by the downhill simplex method.

The results of the analysis of the orientation errors impact on the position of the guidance errors extrema allow to make the following conclusions:

- Point $(\theta, \phi) \approx\left(79,8^{\circ} ; 100^{\circ}\right)$ is the point of discontinuity of the second kind for MSD of the guidance errors by the azimuth, where $\sigma_{\theta}$ increase greatly;

- the selected method of the extreme search witnessed the instability to the change of the input data regarding the search of the extremal values, that together with the results of the analysis of the graphic results (Fig 5-6) allows to speak about certain areas of minimal and maximal values of MSD errors. 


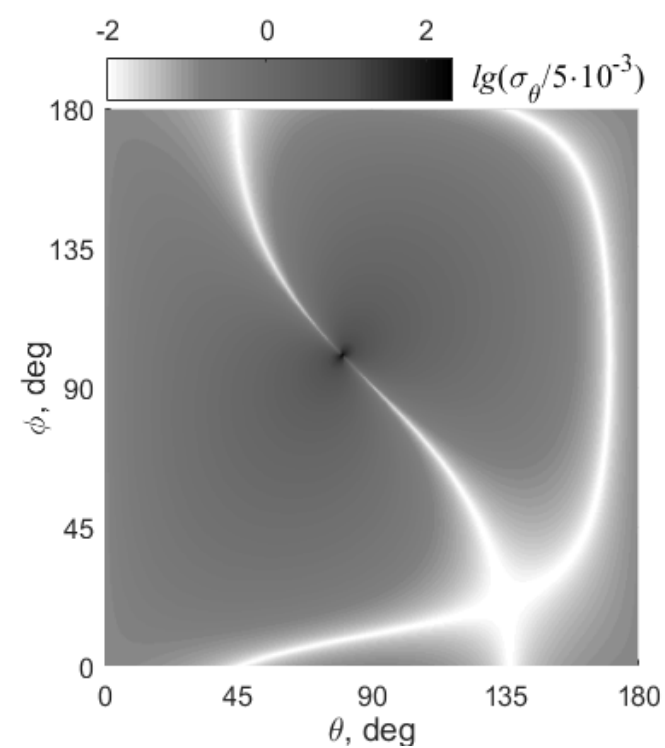

a)

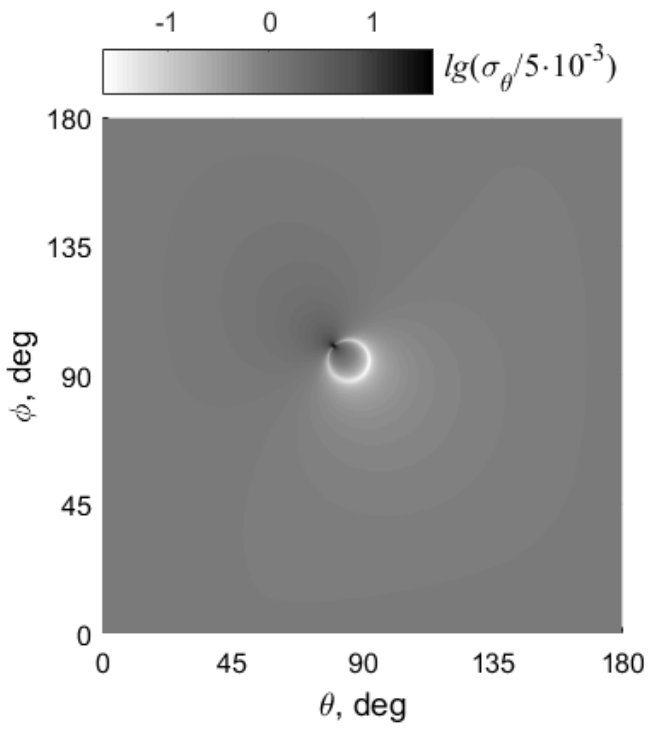

c)

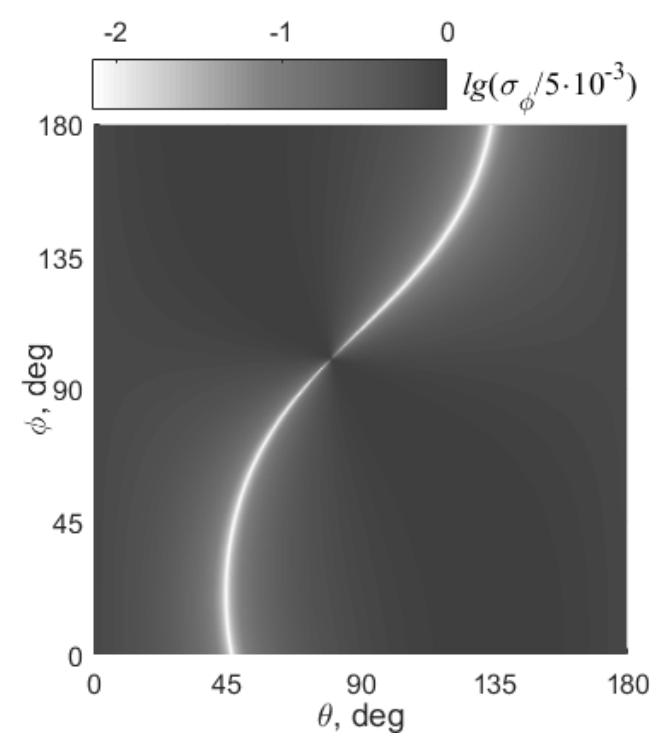

b)

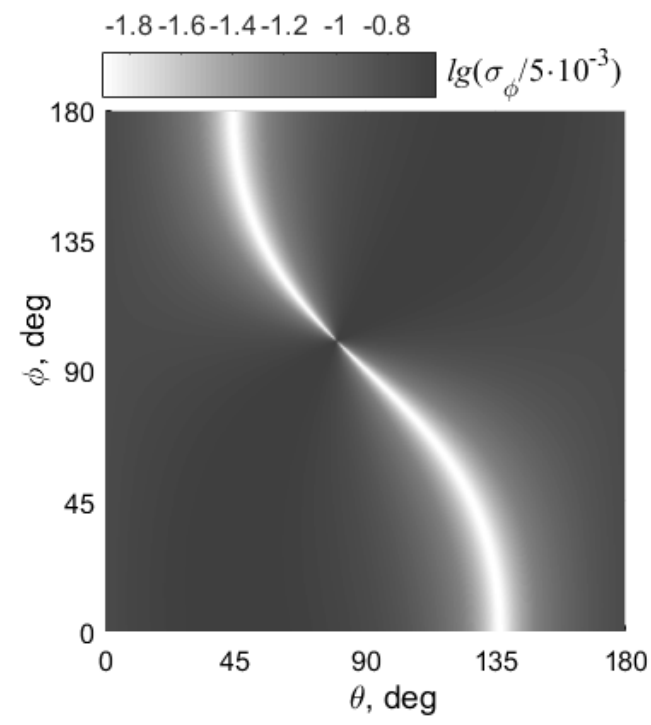

d)

Fig. 6. Separate results of MSD study of guidance errors by the azimuth (a, c ) and elevation angle (b, d ), depending on on the target direction for various ratio of the errors: $\sigma_{\alpha}=5 \cdot 10^{-5} \mathrm{rad}, \sigma_{\beta}=5 \cdot 10^{-5} \mathrm{rad}$,

$$
\sigma_{\gamma}=5 \cdot 10^{-3} \mathrm{rad}(\mathrm{a}, \mathrm{b}) ; \sigma_{\alpha}=5 \cdot 10^{-5} \mathrm{rad}, \sigma_{\beta}=5 \cdot 10^{-3} \mathrm{rad}, \sigma_{\gamma}=5 \cdot 10^{-5} \mathrm{rad}(\mathrm{c}, \mathrm{d})
$$

\section{Conclusions}

The paper formalizes and generalizes mathematical relations and methodical approaches to the determination of the guidance errors dependence on the carrier orientation errors.

As a result of the research, carried out, it is determined:

1. Guidance errors functionally depend on the orientation errors, they cannot be considered to be independent.

2. In the area of the values of the possible mutual location and orientation of the carrier and the target there exist areas of minimal and maximal guidance errors. For the application of the means of the directed action the method of preliminary rational orientation of the carrier can be used for the decrease of the guidance errors.

Development of the methodical and technical recommendations, regarding the minimization of the Scientific Works of VNTU, 2020, № 2 
guidance errors to the acceptable value and avoiding their hitting in the area of maximal values.

\section{REFERENCES}

1. Orbons S. Do Non-Lethal Capabilities License to 'Silence'? / Sjef Orbons // Journal of Military Ethics. - 2010. Vol. 9, Iss. 1. - P. $78-99$.

2. Matlin I. I. Radiolocation : manual for the enlisted persons / I. I. Matlin. - M. : Military Publishing House Voenizdat of the Ministry of Defence of the USSR, 1960. - 443 p. (Rus).

3. Mosienko S. A. Concept of the construction of ground-based robotic missile strike system. Second edition, revised / S. A. Mosienko, V. I. Lokhtin. - M. : Publishing House «SAMPOLIGRAFIST», 2014. - 122 p. (Rus).

4. Momot R. V. Impact of the antennas characteristics on the accuracy of the coordinates determination of the radiation sources / R. V. Momot, G. V. Khudov // Collection of scientific papers of Kharkiv University of Air Forces. - 2013. - Issue 2 (35). - P. 58 - 61. (Ukr).

5. Study of the guidance system and "error map" construction for the telescope ТПЛ-1M on the base of the stars observation / S. I. Ignatovych, Ya. M. Motrunych, I. F. Naibauer [et al.] // Scientific Bulletin of Uzhorod University, Series Physics. - 2008. - Issue 22. - P. 105 - 110. (Ukr).

6. Recommendation ITU-R S. 1064-1 Pointing accuracy as a design objective for earthward antennas On Board Geostationary Satellites in the Fixed-Satellite Service, 1994-1995 [Electronic resource] // Access mode : https://www.itu.int/dms pubrec/itu-r/rec/s/R-REC-S.1064-1-199510-I!!PDF-E.pdf.

7. Telescope Fabra ROA Montsec : A New Robotic Wide Field Baker-Nunn Facility / Octavi Fors, Jorge Núñez, José Luis Muiños [et al.] // Publications of the Astronomical Society of the Pacific. - 2013. - Vol. 125, № 927. - P. 522 - 538. DOI: 10.1086/670941.

8. First Scientific VLBI Observations Using New Zealand 30 Meter Radio Telescope WARK30M / L. Petrov, T. Natusch, S. Weston [et al.] // Publications of the Astronomical Society of the Pacific. - 2015. - Vol. 127, № 952. P. 516 - 522. DOI: $10.1086 / 681965$.

9. Theoretical fundamentals of radiolocation / [A. A. Korostelev, N. F. Kluev, Yu. A. Melnyk et al .] ; under the editorship of V.E. Dudkevych. - M. : Soviet Radio, 1978. - 608 p. (Rus).

10. Mamon V. A. Ballistic support of the flights / V. A. Mamon. - L. : A. F. Mozhayskyi Military Space Engineering University (MSEU), 1990. - 623 p. (Rus).

11. Ammeral L. Programming Principles in computer graphics / L. Ammeral ; Translated from English by V. A. Lvov. - M. : "Sol System", 1992. - 224 p. (Rus).

12. Rapport L. B. Method of determination of relative orientation / L. B. Rapport // Control problems. - 2010. Issue 5. - P. 57 - 64. (Rus).

13. ISO 11606:2000. Ships and marine technologies. Marine electromagnetic compasses, 2000 [Electronic resource] // Access mode : http://www.sis.se/document/preview\&usg=AOvVaw0W1jTaR7Qj13TNot6gX170.

14. Ventsel E. S. Probability theory : Textbook - for higher education establishments. - 6-edition, stereotyped. / E. S. Ventsel. - M. : High School, 1999. - 576 p. (Rus).

Editorial office received the paper 12.06.2020.

The paper was reviewed 20.06.2020.

Biloborodov Oleg - Cand. Sc. (Eng.), Postdoctoral Student, Military Unit A4566, spe- ciality 05.13.06information technologies.

Central Scientific-Research Institute of the Armaments and Military Equipment of the Armed Forces of Ukraine, Military Unit A4566. 